\title{
Microparticle manipulation using inertial forces
}

\author{
Michael Eglin \\ Department of Engineering Physics, University of Wisconsin, Madison, Wisconsin 53706
}

Mark A. Eriksson

Department of Physics, University of Wisconsin, Madison, Wisconsin 53706

Robert W. Carpick ${ }^{\text {a) }}$

Department of Engineering Physics, University of Wisconsin, Madison, Wisconsin 53706

(Received 1 July 2005; accepted 19 December 2005; published online 2 March 2006)

\begin{abstract}
We demonstrate controlled manipulation of large quantities of microparticles on a surface using inertial forces. Motion is induced by applying a periodic parabolic wave form to a shear-polarized piezoelectric plate coupled to a substrate on which the particles reside. Particles move in steps of 10 to $50 \mathrm{~nm}$ per cycle, and the particle motion is mass selective. Particle velocity is varied by changing the frequency of the wave form. Calculated inertial forces acting on the particles correspond closely to friction forces between individual microparticles and the substrate, as measured by coupling an individual particle to an atomic force microscope. The results provide insight into the characteristics of particle-surface interactions, and demonstrate the potential for controlled manipulation and separation of large collections of particles without the need for a fluid medium. ( 2006 American Institute of Physics. [DOI: 10.1063/1.2172401]
\end{abstract}

Understanding and controlling the motion of small particles is critical for micro- and nanoassembly, ${ }^{1}$ microfluidics, ${ }^{2}$ including biological and colloidal science applications, chemical mechanical polishing, ${ }^{3}$ and xerographic processes. ${ }^{4}$ However, a method for deterministic transport of large quantities of particles without a carrier medium does not exist.

Inertial forces are one candidate for such manipulation. The use of inertial forces to create dynamic motion was first demonstrated by Pohl ${ }^{5}$ and is used today for coarse positioning in commercial scanning probe instruments. ${ }^{6,7}$ Recently, inertial transport of microliter droplets $(0.5-5 \mathrm{~mm}$ in diameter) on a hydrophobic substrate using asymmetric lateral vibrations was demonstrated. ${ }^{8}$ Here we present the use of thin piezoelectric plates to generate in-plane displacements of substrates to manipulate solid spherical particles as small as $60 \mu \mathrm{m}$ in diameter by inertial forces. We present an analytical model describing the inertial forces on the particles and verify it by measuring the friction force between the substrate and particles directly attached to an atomic force microscope (AFM) cantilever.

Stainless steel or glass particles (Duke Scientific Corporation, Palo Alto, CA) are placed on a substrate glued to a shear polarized piezoelectric plate (type EBL1, Staveley Sensors Inc., East Hartford, CT) [Fig. 1(a)]. A drive signal, generated by an arbitrary wave form generator (33250A, Agilent, Palo Alto, CA) and amplified by a high frequency amplifier (HSA4101, NF Corporation, Yokohama, Japan) is applied to the piezo. With the drive signal $U(t)$ chosen to be a repeated parabolic signal [Fig. 1(a), inset], the surface displacement $x(t)$ is the following:

$$
x(t)=d_{15} U(t)=d_{15}\left(8 U_{m} f_{d}^{2} t^{2}-U_{m}\right)
$$

for $t \in\left[\left(-1 / 2 f_{d}\right),\left(1 / 2 f_{d}\right)\right]$, and its periodic continuation outside this interval. $d_{15}$ is the piezoelectric coefficient, $f_{d}$ is the

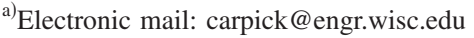

drive frequency, and $U_{m}$ is the drive amplitude. During the smooth parabolic part of the displacement, the particles move laterally with the substrate at a constant, low acceleration. At the cusp of the wave form, the direction of the surface displacement is suddenly reversed and the particles, due to their inertia, continue sliding a small distance. Figures 1(b) and 1(c) show topview images from a movie of stainless steel particles moving on a silicon surface, where the piezo was driven repeatedly with this wave form. The images were captured with a digital camera attached to an optical microscope. ${ }^{9}$ Figure 1(b) shows the particles' initial positions, and Fig. 1(c) shows their positions after applying a wave form with an amplitude of $\pm 71 \mathrm{~V}$ at $10 \mathrm{kHz}$ for $0.75 \mathrm{~s}$. Figure 1(c) also indicates the original positions of the particles and their trajectories, demonstrating that all particles travel in the same direction. Figure 1(d) is a stack of colorshaded images captured in between the frames shown in Figs. 1(b) and 1(c), illustrating the progressive motion of the particles. From higher magnification movies it is obvious that no rolling is involved in the particle motion.

The direction of the motion is controlled by the polarity of the applied drive signal. Reversing the polarity causes the particles to move in the opposite direction, as shown in the full movie. ${ }^{9}$ Two-dimensional particle manipulation is achieved by stacking two piezos with perpendicular shear directions and applying the drive voltage to each sequentially. Figure 1(e) shows a color-shaded stack of images of particles moving first down and then to the right. The images, also extracted from a movie, demonstrate the twodimensional particle manipulation. ${ }^{10}$

The particle velocity is strongly influenced by the drive frequency. While a wide spread of velocities at a particular frequency is observed, an increase in the maximum velocity from 50 to $600 \mu \mathrm{m} / \mathrm{s}$ for 90 and $200 \mu \mathrm{m}$ diameter glass particles occurs with increasing frequency (Fig. 2). This is due to the increasing number of inertial pushes on the particles per unit time with increasing frequency. The average step sizes are independent of the drive frequency and on the 
(a)
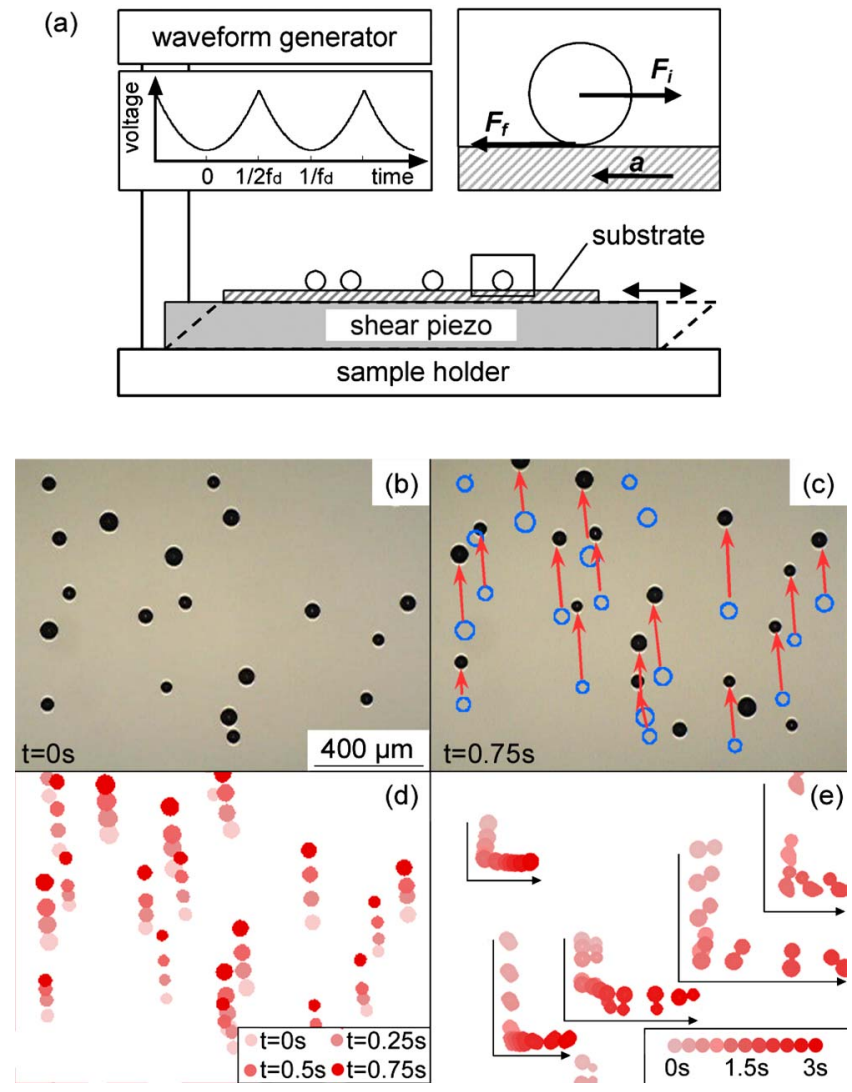

FIG. 1. (Color online) One- and two-dimensional transport of stainless steel particles. (a) Experimental setup: a shear polarized piezoelectric plate is mounted on a steel sample holder. A thin substrate is glued onto the top electrode of the piezo, and particles are placed on it. A periodic parabolic wave form (left inset) is applied to the piezo. The inset on the right shows the forces (inertial $F_{i}$ and frictional $F_{f}$ ) acting on a particle when the substrate undergoes acceleration $a$. (b)-(e) Images taken from movies illustrating the one- and two-dimensional particle motion. For details see text.

order of $10-50 \mathrm{~nm}$ per step, which indicates that sliding occurs only during the turnaround of the signal, as expected for the inertial mechanism. The spread in velocities and the fact that some particles remain stuck to the surface after the actuation begins is most likely due to surface imperfection of the particles and the substrate. At frequencies above a certain threshold (12 and $14 \mathrm{kHz}$ for the 200 and $90 \mu \mathrm{m}$ particles, respectively), the particle velocities are reduced. At these frequencies, the inertial forces during the smooth parabolic part of the wave form exceed the particle-substrate friction force. This creates motion in the direction opposite that intended, decreasing the mean particle velocity. If the drive frequency is increased further, random motion of the particles occurs.

The actual surface displacement of the silicon substrate and the particle-substrate friction force is measured with glass particles attached to AFM cantilevers (so-called colloidal probes ${ }^{11}$ ). $70 \mu \mathrm{m}$ glass particles are glued to commercial AFM cantilevers with an UV curable epoxy resin (NOA61, Norland Products, NJ) using a home-built micromanipulation stage. The cantilever spring constants are calibrated in normal and torsional mode with the added mass method. ${ }^{12,13}$ The surface displacement during actuation of the piezo is measured by bringing the colloidal probe into contact with the substrate with its long axis perpendicular to the shear direction of the piezo. A drive voltage small enough to prevent slip of the particle is applied to the piezo and the surface displacement is calculated from the lateral deflection signal Downloaded 07 Mar 2007 to 128.104.198.190. Redistribution subje

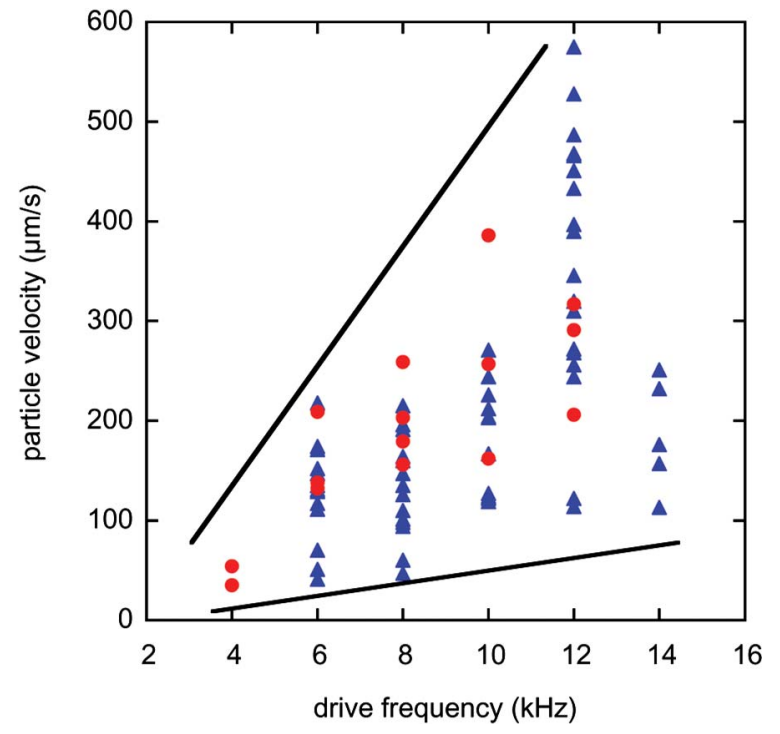

FIG. 2. (Color online) Velocity of glass particles. The velocity of glass particles $(200 \mu \mathrm{m}, \boldsymbol{\Delta} 90 \mu \mathrm{m})$ is shown as a function of the drive frequency on a silicon surface with a parabolic wave form (amplitude of $\pm 71 \mathrm{~V}$ ). The maximum and minimum velocity of the particles increases with increasing frequency. The lines are added as a guide for the eye.

of the AFM. During these experiments, scanning of the AFM scanning piezo itself is turned off. Figure 3 shows the drive voltage (a), the surface displacement (b), and the surface acceleration (c) (calculated from (b) after smoothing the data with a running average). The maximum displacement occurs at time $t_{1}$ [Fig. 3(a)], approximately $10 \mu \mathrm{s}$ after the maximum in the drive signal. There is also a broadening of the peak to a width $\Delta t$ and thus a reduction of the maximum acceleration. These effects are due to hysteresis which is inherent to piezoelectric materials, and this limits the inertial force that can be applied.

The inertial force $F_{i}$ on the particle at the cusp is calculated by $F_{i}=m a$ with $m$ being the particle mass and $a$ the surface acceleration. If we model the acceleration as constant during the time period $\Delta t$ at the peak of the displacement [Fig. 3(b)], it can be approximated by $a=\Delta \nu / \Delta t$. $\Delta t$ is measured from Fig. 3(b) and found to be $15 \mu$ s for all drive frequencies and amplitudes used in our experiments. The change in surface velocity is estimated by $\Delta \nu=\nu_{1}-\nu_{2}$, with $\nu_{1}$ and $\nu_{2}$ the velocities at the beginning and end of the time period $\Delta t$ [Fig. 3(b)]. The velocities are calculated from Eq. (1). The inertial force acting on a particle with diameter $D$ and density $\rho$ is

$$
F_{i}=m \frac{\nu_{1}-\nu_{2}}{\Delta t}=\frac{8 f_{d} d_{15} U_{m} D^{3} \pi \rho}{3 \Delta t} .
$$

To estimate the inertial forces necessary to move particles placed on a substrate, we slowly increase the drive amplitude, and observe the amplitude at which the particles start to move. In three independent experiments with $70 \mu \mathrm{m}$ glass particles, with a parabolic drive of $12 \mathrm{kHz}$, the first particle motion is observed at $19 \pm 6 \mathrm{~V}$, which according to Eq. (2) equals $54 \pm 17 \mathrm{nN}$. At full drive amplitude $(71 \mathrm{~V}$, or $200 \mathrm{nN})$ $80 \% \pm 7 \%$ of all particles are observed to move.

These forces are compared to friction forces measured for individual particles with the colloidal probe AFM. ${ }^{14}$ Friction is measured from the first moment of contact between the particle and the surface (Fig. 4), with only a small normal to AlP license or copyright, see http://apl.aip.org/apl/copyright.jsp 


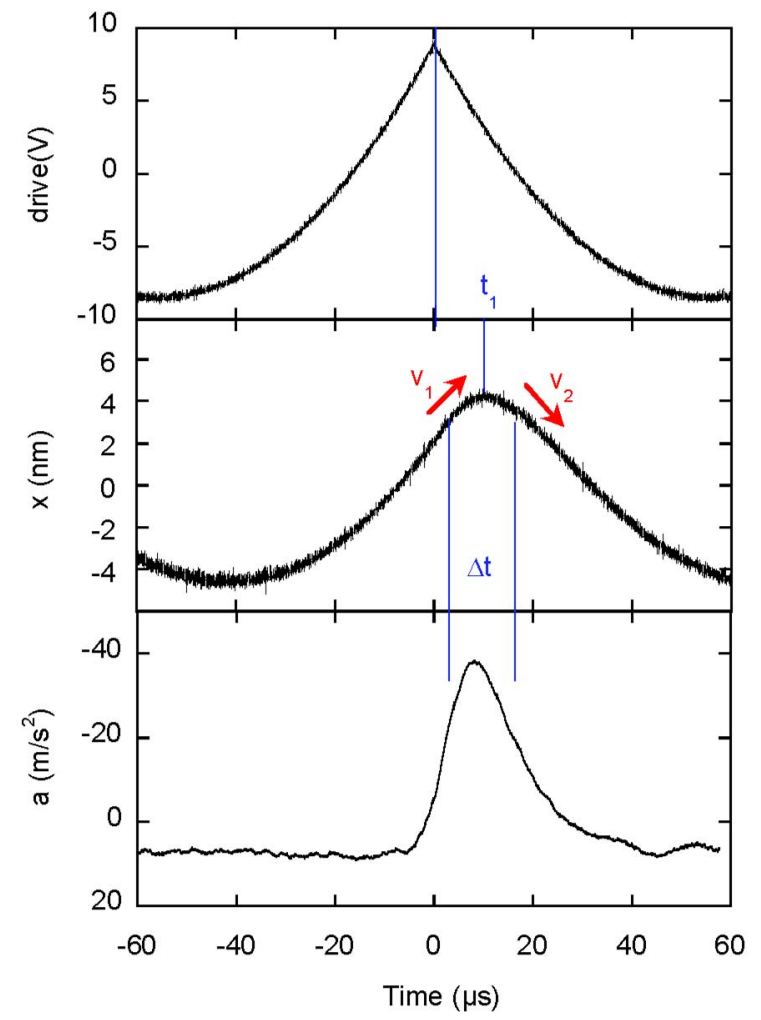

(a)

(b)

FIG. 3. (Color online) Drive voltage, surface displacement, and surface acceleration. (a) Drive voltage applied to the piezo (parabolic wave form, 10 V, $9 \mathrm{kHz}$ ). (b) Surface displacement measured with an AFM. (c) Surface acceleration calculated from (b) after smoothing with a running average.

load of $1.0 \pm 0.5 \mathrm{nN}$ besides the additional $45 \pm 13 \mathrm{nN}$ of adhesion acting. After a rapid increase during contact formation, a slow increase with sliding distance occurs, which levels off after $\sim 100 \mu \mathrm{m}$ of sliding. Two separate measurements with individually prepared cantilevers with $70 \mu \mathrm{m}$ particles attached exhibit initial friction forces of 25 and $45 \mathrm{nN}$, respectively, and the forces level off at 40 and $140 \mathrm{nN}$, respectively. These forces correspond well to the inertial force of $54 \pm 17 \mathrm{nN}$ at which the first particles start to move on the actuated substrate, and the upper limit of these forces is in the range where a large fraction of the particles move on the substrate. The spread of the friction forces for the two particles shows that friction is dependant on the particular particle, which has also been observed in singleparticle adhesion measurements by Heim et al. ${ }^{15}$ This is consistent with our observation that under the same drive conditions some particles move while others do not, which as stated above is likely due to surface imperfections, which indeed are observed in electron micrographs of the particles' surfaces.

We demonstrate a way to induce motion of large numbers of microparticles on a surface with no carrier medium. The method is simple and versatile, with the only prerequisite that the combination of particle mass and substrate acceleration must produce enough inertia to overcome the friction force of the particle/substrate interface. By coupling an AFM directly to individual glass particles, we measure the friction force between the particles and the substrate, and

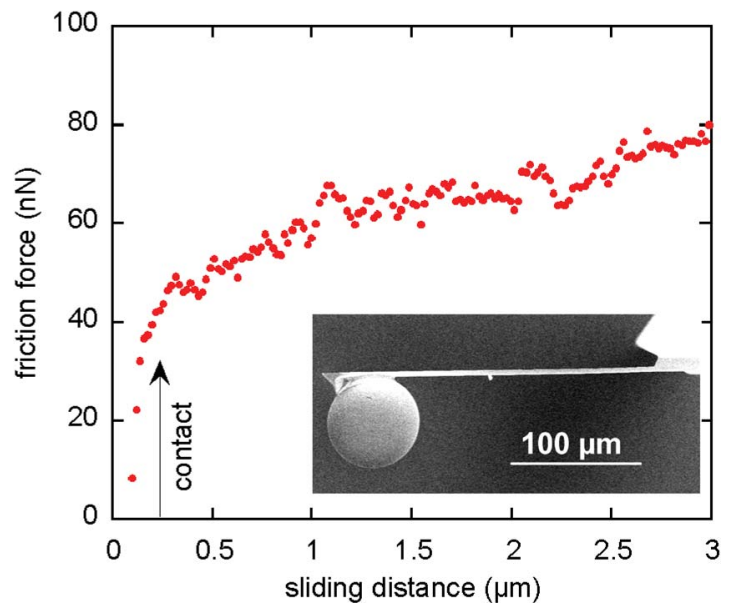

FIG. 4. (Color online) Friction for a $70 \mu \mathrm{m}$ glass particle. The friction force between a glass particle and a silicon surface. The colloidal AFM probe is shown in the inset.

find good agreement between a simple analytical model and the measured behavior of the actuated particles. A further reduction of the particle size being moved can be achieved by the use of piezoelectric materials with lower hysteresis to increase the surface acceleration or by reducing frictional forces between particles and the substrate.

The authors acknowledge Dr. In-Ha Sung for preparing the colloidal probes, and T. Swenson and C. Torres for assistance with sample preparation, piezocharacterization, and data acquisition. This work was supported by The U.S. Department of Defense, Army Research Office, Chemical Sciences Division, under Contract No. DAAD19-03-1-0102, and by the NSF under Grant Number DMR-0094063.

${ }^{1}$ G. M. Whitesides and B. Grzybowski, Science 295, 2418 (2002).

${ }^{2}$ A. Terray, J. Oakey, and D. W. M. Marr, Science 296, 1841 (2002).

${ }^{3}$ L. M. Cook, J. Non-Cryst. Solids 120, 152 (1990).

${ }^{4}$ H. Mizes, M. Ott, E. Eklund, and D. Hays, Colloids Surf., A 165, 11 (2000).

${ }^{5}$ D. W. Pohl, Rev. Sci. Instrum. 58, 54 (1987).

${ }^{6}$ Q. Dai, R. Vollmer, R. W. Carpick, D. F. Ogletree, and M. Salmeron, Rev. Sci. Instrum. 66, 5266 (1995).

${ }^{7}$ K. Besocke, Surf. Sci. 181, 145 (1987).

${ }^{8}$ S. Daniel, M. K. Chaudhury, and P.-G. De Gennes, Langmuir 21, 4240 (2005).

${ }^{9}$ See EPAPS Document No. E-APPLAB-88-041606 (http://ftp.aip.org/cgibin/epaps? ID=E-APPLAB-88-041606) for a movie showing one dimensional particle manipulation. For more information on EPAPS, See http:// www.aip.org/pubserves/epaps.html

${ }^{10}$ See EPAPS Document No. E-APPLAB-88-041606 (http://ftp.aip.org/cgibin/epaps? ID=E-APPLAB-88-041606) for a movie showing two dimensional particle manipulation. For more information on EPAPS, See http:// www.aip.org/pubserves/epaps.html

${ }^{11}$ W. A. Ducker, T. J. Senden, and R. M. Pashley, Nature (London) 353, 239 (1991).

${ }^{12}$ J. P. Cleveland, S. Manne, D. Bocek, and P. K. Hansma, Rev. Sci. Instrum. 64, 403 (1993).

${ }^{13}$ C. P. Green, H. Lioe, J. P. Cleveland, R. Proksch, P. Mulvaney, and J. E. Sader, Rev. Sci. Instrum. 75, 1988 (2004).

${ }^{14}$ S. Ecke, R. Raiteri, E. Bonaccurso, C. Reiner, H. J. Deiseroth, and H. J. Butt, Rev. Sci. Instrum. 72, 4164 (2001).

${ }^{15}$ L. O. Heim, S. Ecke, M. Preuss, and H. J. Butt, J. Adhes. Sci. Technol. 16 829 (2002). 